\title{
ARTICLES
}

\section{Wave competition in excitable modulated media}

\author{
I. Sendiña-Nadal, ${ }^{1, *}$ M. Gómez-Gesteira, ${ }^{1}$ V. Pérez-Munuzuri, ${ }^{1}$ V. Pérez-Villar, ${ }^{1}$ J. Armero, ${ }^{2}$ L. Ramírez-Piscina, ${ }^{3}$ \\ J. Casademunt, ${ }^{2}$ F. Sagués, ${ }^{4}$ and J. M. Sancho ${ }^{2}$ \\ ${ }^{1}$ Grupo de Física Non Lineal, Facultade de Física, Universidade de Santiago de Compostela, 15706 Santiago de Compostela, Spain \\ ${ }^{2}$ Departament d'Estructura i Constituents de la Matèria, Universitat de Barcelona, Avenida Diagonal 647, \\ E-08028 Barcelona, Spain \\ ${ }^{3}$ Departament de Física Aplicada, Universitat Politècnica de Catalunya, Avenida Dr. Gregorio Marañón 50, \\ E-08028 Barcelona, Spain \\ ${ }^{4}$ Departament de Química Física, Universitat de Barcelona, Avenida Diagonal 647, E-08028 Barcelona, Spain
} (Received 15 April 1997; revised manuscript received 19 June 1997)

\begin{abstract}
The propagation of an initially planar front is studied within the framework of the photosensitive BelousovZhabotinsky reaction modulated by a smooth spatial variation of the local front velocity in the direction perpendicular to front propagation. Under this modulation, the wave front develops several fingers corresponding to the local maxima of the modulation function. After a transient, the wave front achieves a stationary shape that does not necessarily coincide with the one externally imposed by the modulation. Theoretical predictions for the selection criteria of fingers and steady-state velocity are experimentally validated. [S1063-651X(97)02812-2]
\end{abstract}

PACS number(s): 05.70.Ln, 03.40.Kf

During the past few decades, the Belousov-Zhabotinsky (BZ) reaction has been the paradigm of nonlinear active media $[1,2]$ due to its suitability both to mimic some of the properties observed in more complex systems such as cardiac tissue or neural networks $[3,4]$ and to validate theoretical predictions on autowave behavior [5-7]. Thus the properties of different autowaves such as spirals [4,8-13], superspirals [14], V-shaped patterns $[5,15]$, pinwheels $[16,17]$ and targets waves $[6,18]$ have been widely treated in the literature.

On the other hand, velocity and shape selection in pattern forming interfaces have been issues of central interest in recent years, not only in chemical systems but in a wide range of situations such as viscous fingering, dendritic growth, directional solidification, or flame propagation $[19,20]$. In particular, analytical [21] and experimental [22,23] studies have been carried out recently in inhomogenous media, where a stable front develops a stationary structure as a consequence of the spatial modulation of an external control parameter.

The aim of this paper is to show experimentally the propagation of an initially planar front in an inhomogeneous medium in order to compare its stationary shape and velocity with the one analytically obtained by perturbative techniques in [21] for a general reaction-diffusion system. Experiments were performed by using a photosensitive version of the BZ reaction. In this reaction-diffusion system, the catalyst ruthenium bipyridil $\left(\mathrm{Ru}(\text { bby })_{3}\right)$ presents two states of oxidation that play a part in the reduction-oxidation process by promoting the production of the activator $\left(\mathrm{HBrO}_{2}\right)$ and the inhibitor $\left(\mathrm{Br}^{-}\right)$species. In an illuminated $\mathrm{BZ}$ reaction the excited $\mathrm{Ru}^{2+}$ produces extra $\mathrm{Br}^{-}$, which decreases the excitability of the system and consequently the propagation velocity of au-

\footnotetext{
*Electronic address: irene@fmmeteo.usc.es
}

towaves $[18,24]$. In this way, it is possible to control the excitability of the system by changing the intensity of the applied illumination. In order to avoid convection effects, the catalyst $\mathrm{Ru}(\text { bby })_{3}(0.71 \mathrm{mM})$ was immobilized in a silica-gel matrix in a Petri dish (a gel $1 \mathrm{~mm}$ thick and $14 \mathrm{~cm}$ in diameter was prepared as in Ref. [25]). The reactant concentrations were $0.18 M \mathrm{NaBr}, 0.34 M$ malonic acid, $0.38 M$ $\mathrm{NaBrO}_{3}$, and $0.48 M \mathrm{H}_{2} \mathrm{SO}_{4}$. The solution was poured into the Petri dish at room temperature $\left(23 \pm 1{ }^{\circ} \mathrm{C}\right)$. Experiments were performed for approximately $\frac{1}{2} \mathrm{~h}$ to keep unchanged reactant concentrations. The Petri dish was illuminated from below by a halogen lamp, whose intensity was controlled by means of an external variable resistor and measured by a photometer. Recording was done with a vertically placed charge coupled device camera via an interference filter at $450.6 \mathrm{~nm}$. The images were digitized by an imageacquisition card and analyzed on a personal computer.

The gel was inhomogeneously illuminated by placing under the Petri dish a transparency where the modulation function (pattern from now on) was printed out. To calibrate the medium, different patterns corresponding to constant modulation functions (strips) were used in order to have different illumination conditions. These strips permit measurements of the velocity of a planar wave as a function of the light intensity. Strips were considered to be $0.7 \mathrm{~cm}$ wide to prevent a wave velocity dependence on the strip width [24] and $6 \mathrm{~cm}$ long to allow waves to reach a stationary velocity. This velocity is observed to decrease linearly within the range of light intensities used (Fig. 1).

In order to modulate smoothly the local front velocity in the $x$ direction (waves propagate in the $y$ direction) two different patterns were printed on the transparency $(6.9 \mathrm{~cm}$ wide and $11.7 \mathrm{~cm}$ long). The analytical form of the local front velocity for the two modulation functions $u(x)$ is given by the expression 


\section{$\operatorname{Velocity}(\mathrm{mm} / \mathrm{min})$}

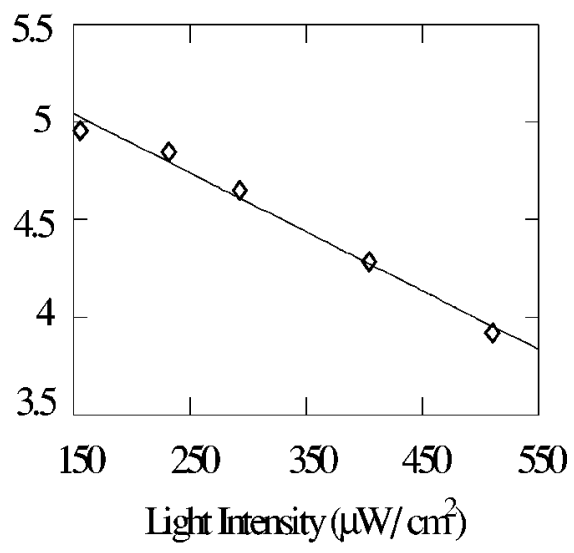

FIG. 1. Dependence of wave velocity on light intensity. The points fit the solid line corresponding to the linear equation: $v(\mathrm{~mm} / \mathrm{min})=c I\left(\mu \mathrm{W} / \mathrm{cm}^{2}\right)+d$, where $c=-3.02 \times 10^{-3}$ and $d$ $=5.50$, with an accuracy greater than $99 \%$.

$$
\begin{aligned}
u(x)= & A_{1} \exp \left(\frac{-\left(s x-x_{1}\right)^{2}}{b_{1}}\right)+A_{2} \exp \left(\frac{-\left(s x-x_{2}\right)^{2}}{b_{1}}\right) \\
& +A_{3} \exp \left(\frac{-\left(s x-x_{3}\right)^{2}}{b_{2}}\right)+A_{4} \cos \left[2 \pi\left(s x-x_{3}\right)\right]+A_{5},
\end{aligned}
$$

where $A_{1}=0.27, A_{2}=0.36, A_{4}=0.18, A_{5}=4.16, s=0.72$, $b_{1}=0.5, b_{2}=0.1, x_{1}=3.5, x_{2}=1.5, x_{3}=2.5$, and $A_{3}=0$ or 0.108 depending on the required modulation function. Both functions, which were obtained using the relationship between planar wave velocity and intensity given by Fig. 1, present five local maxima as shown at the bottom of Fig. 2. The main difference between them is the height of the central maximum. The shortest corresponds to the value of $A_{3}=0$ in the local front velocity $u(x)$ and plotted below Fig. 2(a) and the highest to $A_{3}=0.108$ plotted below Fig. 2(b). A planar wave front, which was induced using a black piece of paper, propagates in the $y$ direction as shown in Figs. 2(a) and 2(b). Each white line in both pictures corresponds to the same wave front at different times (the delay between consecutive lines is $6 \mathrm{~min})$. At an early stage, both waves mimic its modulation function by developing five local maxima (fingers). According to this, the wave fronts evolve towards a five-finger pattern, where each finger moves with different velocity. The dynamics not only depends on the local details of $u(x)$ around the maxima but each finger interacts with its nearest neighbors giving rise to a slow competition process among them. As a result of such an interaction, some of the slowest fingers are eliminated [fingers 1, 3, and 5 in Fig. 2(a) and fingers 1 and 5 in Fig. 2(b)], while some of the fastest ones [fingers 2 and 4 in Fig. 2(a) and fingers 2, 3, and 4 in Fig. 2(b)] survive. After this process, the front attains a stationary shape and velocity, at least during the remaining available experimental time (10 $\mathrm{min})$.

A different perspective of the competition process is shown in Fig. 3, where the three central fingers of the modulation function shown in Fig. 2(a) are plotted in a frame moving at the propagation velocity of the fastest one (the one

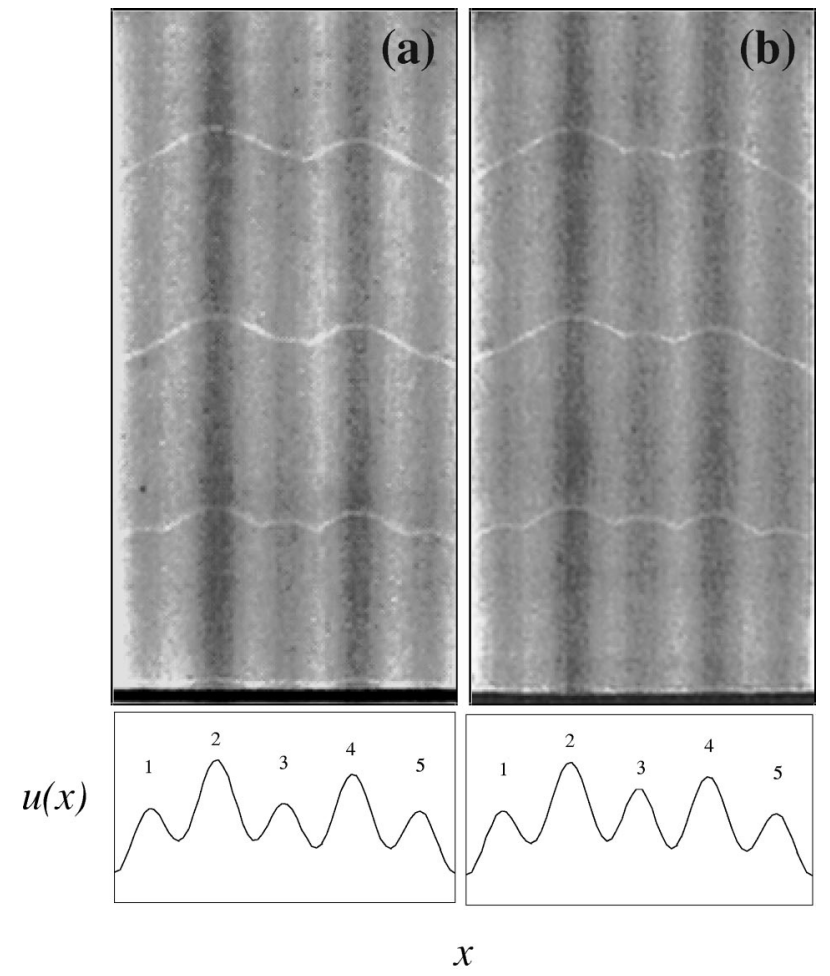

FIG. 2. Evolution of two initially flat fronts under two different spatial modulations of the wave velocity. At early times, the fronts mimic the modulation function with five fingers each. During the transient, fingers 1,3 , and 5 disappear in (a) and only 1 and 5 disappear in (b). Fronts, from bottom to top, corresponding to 0,6 , 12 , and $18 \mathrm{~min}$ after the beginning of the experiments. The dark strip represents the piece of paper that generates the initially planar wave front. The plots on the bottom of each figure represent the $x$ modulation of the local velocity.

on the left). This plot shows how the slow finger is actually invaded by the lateral ones, as if two one-dimensional fronts were propagating laterally into the central area. Note that during the competition process, the maxima of the finger on the right do not stay exactly at the same position because the fronts are plotted in the frame moving at the velocity of the finger on the left.

The experimental results confirm the analytical predictions and the theoretical scenario given in Ref. [21] for generic reaction diffusion systems. There the stationary shape

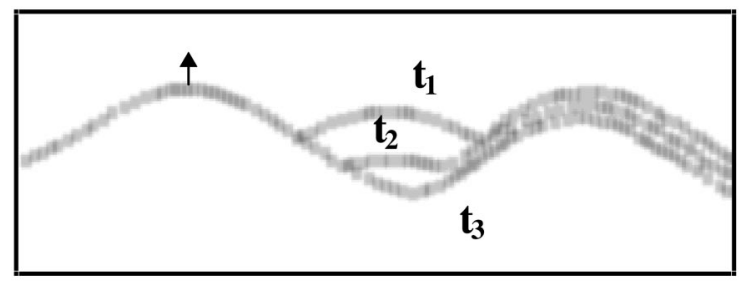

FIG. 3. Invasion of the lateral fast fingers into the area corresponding to the slow central finger taken from Fig. 2(a). The fronts are plotted at three different times $\left(t_{1}=4 \mathrm{~min}, t_{2}=12 \mathrm{~min}\right.$, and $t_{3}$ $=20 \mathrm{~min}$ ) in the reference frame moving at the propagation velocity of the fastest finger (the one on the left). Due to the different velocities of the dominant fingers, the one on the left invades the central area faster than the one on the right. The pattern was substracted from the images to enhance the contrast. 
and velocity of a wave front are predicted as a function of the modulation function $u(x)$ by invoking singular perturbation techniques. The theoretical approach is based on the assumption that the modulation is sufficiently smooth and in practice its period sufficiently large to guarantee the validity of the local equation for the normal velocity $v_{n}$ expressed in terms of the prescribed modulation and the front curvature $\kappa[26-$ 29],

$$
v_{n}=u(x)+D \kappa,
$$

where $D$ is an appropriate diffusion coefficient. As far as this eikonal equation is a faithful approximation of the original problem, the front dynamics must be viewed as strictly local. Accordingly, the competition process does not result from screening of a diffusive field by the fingers but through the dynamics of the contacts between adjacent fingers. The motion of these contacts can in turn be described as lateral fronts propagating in the $x$ direction. The final conclusion is that the number of surviving fingers (i.e., the number of maxima on the stationary front shape) is given by a simple comparison between the local maxima of $u(x)$ and the actual selected velocity $v$. The latter can in turn be estimated as the largest value of [21]

$$
v=u_{m}\left(1-\frac{D}{u_{m}} \sqrt{\frac{\left|u_{m}^{\prime \prime}\right|}{u_{m}}}\right)
$$

evaluated at the different local maxima of $u(x)$. In other words, Eq. (3) taken at the absolute maximum of $u(x)$, denoted $u_{M}$, establishes a sort of minimum finger velocity, below which fingers are annihilated, and thus constitutes a selection rule for finger propagation. Although the dynamical process is local, this selection criterion may be considered as nonlocal, in the sense that the fate of each finger depends on the comparison with all other fingers. In passing, note that the equation above clearly identifies the actual perturbation parameter of the whole analysis as the ratio of two characteristic length scales respectively given by the diffusion length $L_{D}=D / u_{M}$ and the one associated with the modulation $L_{m}^{-1}=\left(\left|u_{M}^{\prime \prime}\right| / u_{M}\right)^{1 / 2}$.

In Fig. 4 the two modulation functions were superimposed (with five local maxima for each one and the central maximum slightly different). Due to the similarity between both functions around the absolute maximum, the theoretical $\left(v_{\text {theor }}\right)$ and experimental $\left(v_{\text {expt }}\right)$ values of $v$ are independent of $A_{3}$ for the considered values given above. The diffu-

\section{$\operatorname{Velocity}(\mathrm{mm} / \mathrm{min})$}

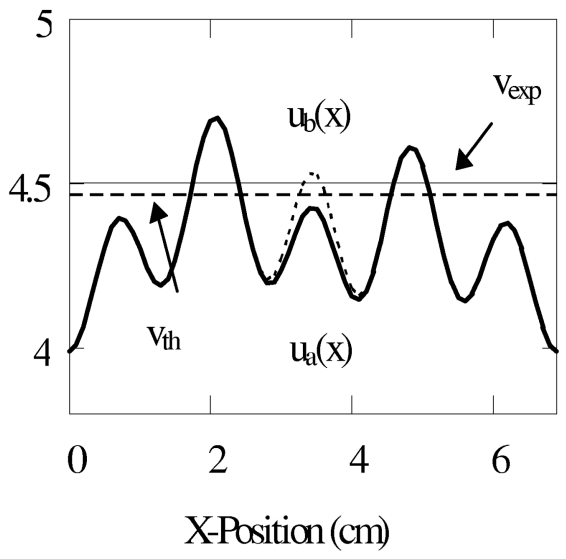

FIG. 4. Superposition of the two modulation functions $u_{a}(x)$ (continuous curve) and $u_{b}(x)$ (dotted curve) given on the bottom of Fig. 2. The straight lines represent the theoretical $v_{\text {theor }}$ (dashed line) and experimental $v_{\text {expt }}$ (continuous line) stationary velocities. A criterion for selection is established: Only those fingers whose maximum local velocity is larger than the final velocity of the stationary front will survive. In both cases, fingers 2 and 4 have a maximum local velocity higher than the one corresponding to the stationary front. Finger 3 only verifies the criterion to be selected in the case of $u_{b}(x)$.

sion coefficient used in Eq. (3) was $D=4 \times 10^{-5} \mathrm{~cm}^{2} / \mathrm{s}$ and was estimated following a procedure similar to the one described in [30], which fits in with the values given in [22]. It can be observed in Fig. 4 how fingers 2 and 4 always survive because their maxima local velocities are larger than $v$ (both theoretically and experimentally). On the other hand, finger 3 only persists when its velocity is given by the modulation function $u_{b}(x)$ (dotted line). This is in good qualitative agreement with Fig. 2(a), where only fingers 2 and 4 survive [corresponding to the modulation function $u_{a}(x)$ ], and fingers 2, 3, and 4 in Fig. 2(b) for $u_{b}(x)$. Moreover, given the lowest-order nature of the theoretical prediction, the quantitative agreement between $v_{\text {theor }}$ and $v_{\text {expt }}$ is satisfactory, the small differences arising probably from unavoidable experimental inaccuracies (light dispersion, etc.).

This work was partially supported by the Comisión Interministerial de Ciencia y Tecnologia (Spain) under Projects Nos. DGICYT-PB94-0623, PB93-759, and PB93-769.
[1] A.N. Zaikin and A.M. Zhabotinskii, Nature (London) 225, 533 (1970).

[2] A.T. Winfree, Science 181, 937 (1973).

[3] J.D. Murray, Mathematical Biology (Springer-Verlag, New York, 1989).

[4] V.I. Krinsky, in Selforganization: Autowaves and Structures Far From Equilibrium (Springer, Berlin, 1984), p. 9.

[5] V. Pérez-Muñuzuri, M. Gómez-Gesteira, A.P. Muñuzuri, V.A. Davydov, and V. Pérez-Villar, Phys. Rev. E 51, 845 (1995).
[6] P. Foerster, S.C. Müller, and B. Hess, Science 241, 685 (1988).

[7] M. Gómez-Gesteira, J.L. del Castillo, V. Pérez-Muñuzuri, M.E. Vázquez, and V. Pérez-Villar, Phys. Rev. E 50, 4646 (1994).

[8] V.I. Krinsky and K.I. Agladze, Physica D 8, 50 (1983).

[9] K.I. Agladze, V.I. Krinsky, and A.M. Pertsov, Nature (London) 308, 834 (1984).

[10] S.C. Müller, T. Plesser, and B. Hess, Physica D 24, 71 (1987). 
[11] A.P. Muñuzuri, M. Gómez-Gesteira, V. Pérez-Muñuzuri, V.I. Krinsky, and V. Pérez-Villar, Phys. Rev. E 50, 4258 (1994).

[12] M. Ruiz-Villarreal, M. Gómez-Gesteira, C. Souto, A.P. Muñuzuri, and V. Pérez-Villar, Phys. Rev. E 54, 2999 (1996).

[13] M. Ruiz-Villarreal, M. Gómez-Gesteira, and V. Pérez-Villar, Phys. Rev. Lett. 78, 779 (1997).

[14] V. Pérez-Muñuzuri, R. Aliev, B. Vasiev, V. Pérez-Villar, and V.I. Krinsky, Nature (London) 353, 740 (1991).

[15] V. Pérez-Munuzuri, C. Souto, M. Gómez-Gesteira, A.P. Muñuzuri, V.A. Davydov, and V. Pérez-Villar, Physica D 94, 148 (1996).

[16] N. Kreisberg, W.D. McCormick, and H.L. Swinney, J. Chem. Phys. 91, 6532 (1989).

[17] X. Noszticzius, W. Horsthemke, W. D. McCormick, H.L. Swinney, and W.Y. Tam, Nature (London) 329, 619 (1987).

[18] A.P. Muñuzuri, V.A. Davydov, M. Gómez-Gesteira, V. Pérez-Muñuzuri, and V. Pérez-Villar, Phys. Rev. E 54, 1 (1996).

[19] P. Pelcé, Dynamics of Curved Fronts in Perspectives in Physics (Academic Press, New York, 1988).
[20] D.A. Kessler, J. Koplik, and H. Levine, Adv. Phys. 35, 255 (1988).

[21] J. Armero, A.M. Lacasta, L. Ramírez-Piscina, J. Casademunt, J.M. Sancho, and F. Sagués, Europhys. Lett. 33, 429 (1996); J. Armero et al., Phys. Rev. E (to be published).

[22] S.C. Müller, O. Steinbock, and V.S. Zykov, Phys. Rev. E 48, 3295 (1993).

[23] E. Dulos, J. Boissonade, and P. DeKepper, Physica A 188, 120 (1992).

[24] M.K. Ram-Reddy, Zs. Nagy-Ungvarai, and S.C. Müller, J. Phys. Chem. 98, 47 (1994).

[25] T. Yamaguchi, L. Kuhnert, Zs. Nagy-Ungvarai, S.C. Müller, and B. Hess, J. Phys. Chem. 95, 5831 (1991).

[26] W.K. Burton, N. Cabrera, and F.C. Frank, Philos. Trans. R. Soc. London Ser. A 243, 299 (1951).

[27] M.L. Frankel and G.I. Sivashinsky, J. Phys. (Paris) 48, 25 (1987).

[28] K. Kawasaki and T. Ohta, Prog. Theor. Phys. 67, 147 (1982).

[29] V.S. Zykov, Biophysics 25, 906 (1980).

[30] P. Foerster, S.C. Müller, and B. Hess, Proc. Natl. Acad. Sci. USA 86, 6831 (1989). 